\title{
A New Protocol to Evaluate Waterproof Effect of Lip Gloss
}

\author{
Sonia Laneri, Ritamaria Di Lorenzo, Antonia Sacchi and Irene Dini* \\ Department of Pharmacy, Italy \\ *Corresponding author: Irene Dini, Department of Pharmacy, University of Naples Federico II, Italy
}

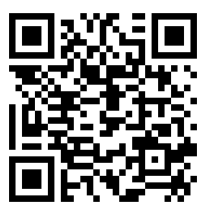

\begin{abstract}
ARTICLE INFO

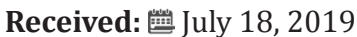

Published: 幽 July 23, 2019

Citation: Sonia Laneri, Ritamaria Di Lorenzo, Antonia Sacchi, Irene Dini. A New Protocol to Evaluate Waterproof Effect of Lip Gloss. Biomed J Sci \& Tech Res 19(5)-2019. BJSTR. MS.ID.003376.

ABSTRACT

A new method to evaluate lip gloss and lipstick waterproof level according to the COLIPA Guidelines for Evaluating Sunscreen Product Water Resistance in 2006 is proposed, moreover the changes in color ( $\mathrm{L}^{*}$ value) by Skin Colorimeter ${ }^{\circledR}$ CL 400 Courage \& Kazama was measured. Tests were carried out on 20 volunteers validating the efficiency of the used method by comparing non-waterproof lip gloss/lipstick and waterproof product results. The results indicated that the lip gloss/lipstick were waterproofs when their mean \% WPR was lower than $50 \%$ and they were removed after two successive immersions in water for 20 minutes at $29^{\circ} \pm 2^{\circ} \mathrm{C}$. Methods used to evaluate lip gloss and lipstick waterproof level has proved effective for assessing the desired goals.
\end{abstract}

Keywords: Lip Gloss; Waterproof Effect; Colorimeter; Percentage Waterproof Ratio (\%WPR)
Abbreviations: WPR\%: Percentage Waterproof Removal Ratio; WR: Water Resistant; SD: Standard Deviation; L*: Value of Color Change; d: Confidence Interval

\section{Introduction}

People use cosmetics to enhance their physical appearance. Cosmetics are broadly classified into basic into Basic make-up, Spot make-up and Point make-up products. The make-up spots include lipsticks, lip gloss and lip pencils, used to enhance and harmonize the shape and size of the lips. A common characteristic of all cosmetic products is better adhesion to the surface on which they are applied. Cosmetics, such as lip gloss and lipstick are utilized to improve the beauty by coating lips to make them more delightful with an attractive color. Moreover, lip gloss and lipstick should ideally be water-resistant while preserving a brilliant coloring Water-resistance or waterproofness are necessary because saliva and atmosphere humidity, which the user is likely to meeting daily, can interact with lip cosmetic products causing their removal.

In addition, the lip gloss and lipstick user would need waterproof products if they want to prevent problems with swimming pool or sea water which would cosmetics alter and/or remove it. A lot of make-up products were declared waterproof, but not much research has been carried out to test waterproof power for makeup products for lips, and those conducted are mainly focused on formulation [1], ingredients, adverse reactions and safety [2]. In 2006, the European Cosmetic, Toiletry and Perfumery Association (COLIPA) issued Guidelines for determining the sun protection factor for water-resistant solar products, measured objectively (COLIPA, 2005.12 and kFDA,2007.05). In this study effective water resistance of seven lip gloss/lipstick sold in beauty shops that affirmed waterproofs on the label was evaluated according to COLIPA Guidelines. Moreover, a lip gloss/lipstick changes in color were analyzed.

\section{Materials and Methods}

\section{Materials}

The analyzed samples were obtained by mixing equivalent quantities of different color shades of the same line (for example, from pink to lilac), uniformly (Figure 1). The comparative analyses of the lip gloss colors before and after immersion in water were reported in (Figures $2 \& 3$ ).

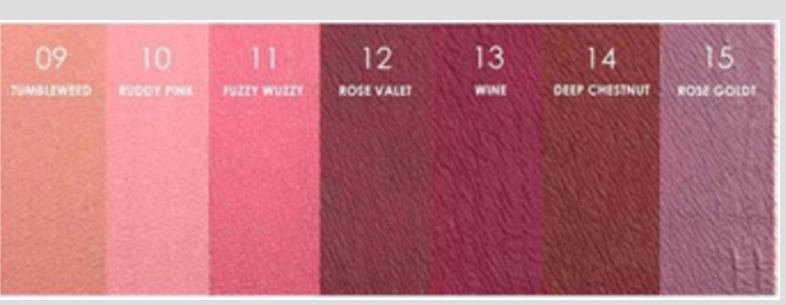

Figure 1: Different nuances of lip gloss mixed in the sample test. 


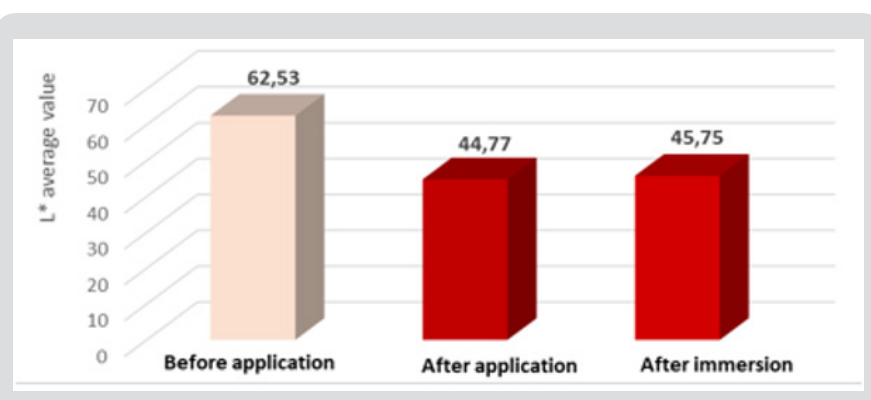

Figure 2: $L^{*}$ average values before, after application and after immersion for $20 \mathrm{~min}$ twice of lip gloss waterproof on the volunteer's forearm.

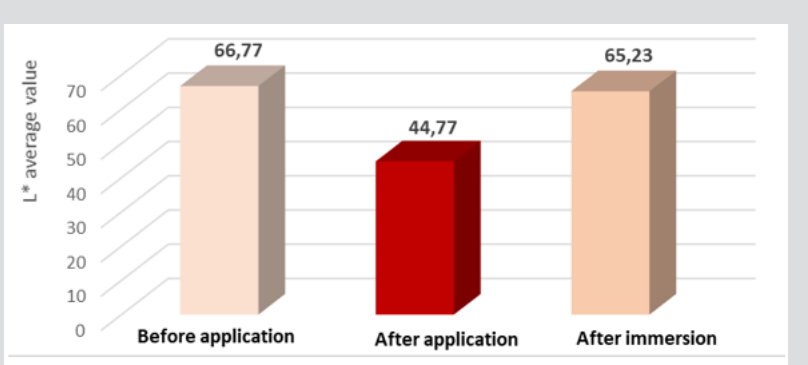

Figure 3: Change of color in $\mathrm{L}^{*}$ average values before, after application and after immersion for 20 min twice of lip gloss does not waterproof on the volunteer's forearm.

\section{Methods}

The study is carried out on the fly part of the 20 female aged between 20 and 65arm. The arm is previously washed, dried and after 20 minutes $12 \pm 0.1 \mathrm{mg}$ of product to be tested was applied on a rectangle drawn with a marker of $6 \mathrm{~cm}^{2}$. The color is measured by a specific colorimeter before application ( $\mathrm{C} 0$ ), 15 minutes after application (C15 ') and after a repeated wash test (Cf), ie immersion in the pool for 40 minutes, $\mathrm{T}^{\circ}$ water $29^{\circ} \pm 2{ }^{\circ} \mathrm{C}(20$ minutes of washing 20 minutes of natural drying, twice). After the WPR\% was calculated (Percentage waterproof removal ratio). If the WPR average percentage was $<50 \%$ the product was considered waterproof.

The \% WPR was calculated as follows: $\%$ WPR $=\frac{\left(C f-C 15^{\prime}\right) \times 100}{\left(C 0-C 15^{\prime}\right)}$

$\mathrm{C} 0=$ value of $\mathrm{L}^{*}$, ie of the individual color before application

$\mathrm{C}^{\prime} 5^{\prime}=$ value of $\mathrm{L}^{*}$, ie of the individual color after 15 minutes from the application of the lip gloss

$\mathrm{Cf}=$ Value of $\mathrm{L}^{*}$, ie of the individual dry color after immersion

The inclusion criteria were:

a. Healthy Caucasian subjects, aged between 20 and 65 years;

b. Sex: female; c. Subjects who have agreed not to apply products other than the one being studied on the test area;

d. Subjects who have agreed not to be exposed to UV for the duration of the study.

The exclusion criteria were:

a. Women who are pregnant or nursing;

b. Subjects with a history of skin hyper-reactivity or intolerance reactions to cosmetic products/ingredients;

c. Subjects with pathologies in the period immediately preceding the study in progress;

d. Subjects undergoing topical or systemic treatment with any drug that may affect the outcome of the test or affected by skin diseases (eczema, psoriasis, lesions, acne);

e. Subjects receiving topical retinoids in the six months prior to the start of the study and for systemic retinoids for the previous 12 months;

f. Subjects who have performed treatments with topical products based on alpha and beta-hydroxy acids in the 45 days prior to the start of the study.

Al the volunteers signed an informed consent before the before starting the study.

\section{Apparatus}

Color measurements were recorded by a Colorimeter CL 400 (Courage \& Khazaka) developed specifically for measuring skin, hair and color make-up color changes [3]. The probe emits white LED light, in a circular way to illuminate the skin uniformly. The light reflected from the skin is measured in the probe and expressed appropriately. The raw data of the probe are corrected with a specific color calculation matrix in order to be correlated to the reference values in the various existing color systems. The color of the measured skin is expressed as XYZ tristimulus values and can be calculated in the $\mathrm{L}^{*} \mathrm{a} * \mathrm{~b} *$ system. Moreover, images obtained by Visiopore ${ }^{\circledR}$ (Courage \& Khazaka) and / or a high resolution Canon Camera (over 12 Megapixel) were used to evaluate the color variation before and after the wash test by photographic comparison.

\section{Statistical Tests}

All statistical tests were performed using SPSS software (version 15). Significance was achieved at a confidence limit of $95 \%$.

\section{Results}

The colorimetric analyses carried out on the arms of 20 volunteers was reported in Table 1. 
Table 1: The mean \%WPR of lip gloss/lipstick evaluated.

\begin{tabular}{|c|c|c|c|c|c|}
\hline & Media \%WPR & SD & t-Student & d & Media \%WPR con limiti di confidenza del 95\% \\
\hline Lip gloss WR & 6.02 & 4.72 & 1.729 & 1.82 & 4.20 \\
\hline Lip gloss no WR & 62,3 & 10,6 & 1,729 & 4,1 & 58,2 \\
\hline Lipstick WR & 5,61 & 3,25 & 1,729 & 1,26 & 2,36 \\
\hline Lipstick no WR & 64,5 & 11,3 & 1,729 & 4,37 & 60,13 \\
\hline
\end{tabular}

\section{Discussion}

The colorimetric analyses showed that the lip gloss and lipstick declared waterproof have an average WPR\% of $4.20 \%$ and $2.36 \%$ with a confidence limit of $95 \%$. These values, $<50 \%$ according the criteria for the waterproof test, indicated these products waterproof. Instead lip gloss and the lipstick declared non-waterproof showed an average WPR\% of $58.2 \%$ and $60.13 \%$, >50\% complying with the statement on the label. The percentage difference in color after the application of lip gloss waterproof on the skin and after the two immersion cycles of 20 minutes $\left(\mathrm{T}=29^{\circ} \pm 2^{\circ}\right)$ was $1.98 \%$, i.e. the color remains on the skin even after washing. The same trend showed the lipsticks waterproof. Instead, when the non-waterproof lip gloss/lipstick were applied, the percentage difference in color after and before was high, so the color does not remain on the skin after washed. Therefore, the efficiency of the used method was validated.

\section{Conclusion}

In this study a new method to evaluate the waterproof claim for lip gloss and lipstick was applied. According to the COLIPA
Guidelines for Evaluating Sunscreen Product Water Resistance our results indicated that the lip gloss/lipstick sold with this claim respected a \% WPR lower than 50\%, while lip gloss/lipstick that did not claimed this property were removed after two successive immersions for 20 minutes at $29^{\circ} \pm 2{ }^{\circ} \mathrm{C}$, therefore, the efficiency of the used method was validated.

\section{References}

1. Westfall A, Sigurdson GT, Giusti MM (2019) Antioxidant, UV Protection, and Antiphotoaging Properties of Anthocyanin-Pigmented Lipstick Formulations. J Cosmet Sci 70(2): 63-76.

2. Van Th Joost, Liem DH, Stolz E (1984) Allergic contact dermatitis to monotertiary-butylhydroquinone in lipgloss. Contact Dermatitis 10(3): 189-190.

3. Matias AR, Ferreira M, Costa P, Neto P (2015) Skin color, skin redness and melanin biometric measurements: comparison study between Antera $(\circledR)$ 3D, Mexameter $(\circledR)$ and Colorimeter $(\circledR)$. Skin Res Technol 21(3): 346-362.

\section{ISSN: 2574-1241}

DOI: 10.26717/BJSTR.2019.19.003376

Irene Dini. Biomed J Sci \& Tech Res

CC (i) This work is licensed under Creative

Submission Link: https://biomedres.us/submit-manuscript.php

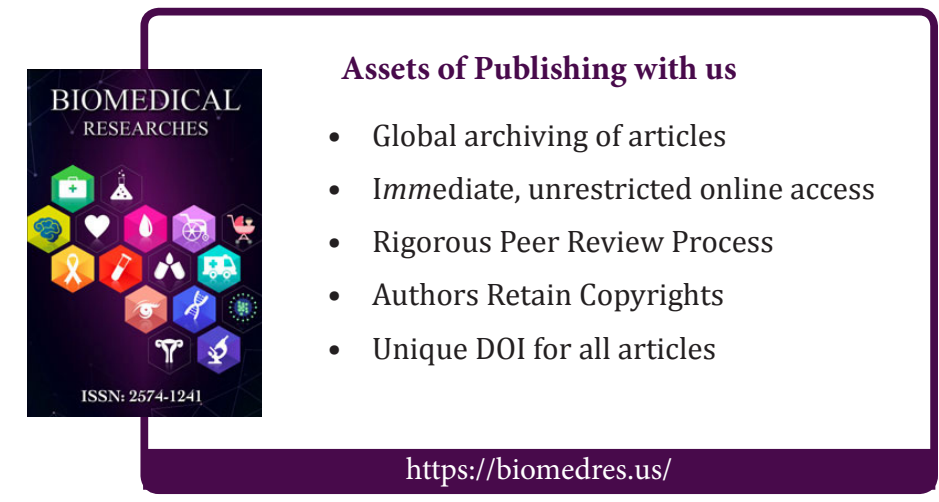

